\title{
Le mosche del capitale: la palinodia del finale
}

\section{Piero dal Bon}

Universitat de Barcelona

dalbon@ub.edu

\begin{abstract}
Il saggio constata come l'ultimo romanzo dell'autore urbinate segni una brusca soluzione di continuità rispetto ai romanzi degli esordi. Mette in relazione questo scarto con il naufragio degli ideali progressisti di riforma del Capitale dall'interno. Documenta come questo mutamento di rotta, allegorizzante, abbia conseguenze su vari livelli della pagina volponiana, a cominciare dalla scomparsa del soggettivismo aspro e espressionistico, che ne contraddistingueva lo stile. Segnala come il registro dominante sia costituito dalla lingua aziendale-burocratica, e rileva il declino di quei procedimenti animistici che caratterizzavano il lirismo eversivo dell'autore.
\end{abstract}

Parole chiave: Volponi, Le mosche del capitale, Allegoria.

\section{Abstract}

The essay examines how the last novel of the author from Urbino is marked by a sudden solution in continuity with respect to his early novels. It places this deviation in the context of the internal collapse of the Capital's progressive reform ideals. The essay documents how this allegorizing change of course had consequences on several levels within Volpini's pages, beginning with the disappearance of the bitter and expressionistic subjectivism, which had distinguished his style. It shows how the dominant register is made up of the language of companies and bureaucracy and reveals the decline of the animistic processes that characterized the author's subversive lyricism.

Key words: Volponi, Le mosche del capitale, Allegory.

Se esperienze prossime all'espressionismo vociano e al neosperimentalismo officinesco sono il vivaio che alimenta e da cui nasce la prima parte dell'opera di Volponi su altri scenari si apre Le mosche del capitale. ${ }^{1}$ L'ultimo romanzo dell'autore è una sorta di dolente palinodia equiparabile per il masochistico

1. D'ora innanzi il romanzo si citerà con la sigla MdC: l'edizione di riferimento è Paolo VOLPONI, Le mosche del capitale, Torino: Einaudi, 1989. 
stravolgimento di ogni positività a quanto Aracoeli rappresenta nel corpo dell'opera della Morante: indugiare su MdC significa da un lato rilevarne il carattere amaramente conclusivo e testamentario, dall'altro rendere più chiaro, per confronto, il senso di un percorso narrativo che dalle esperienze sperimentali degli anni Sessanta e Settanta, fiduciose nel potere extraletterario della propria parola oracolarmente connotata, approda ad un nuova estetica, che estremizza lo sperimentalismo ma facendolo collidere con la constatazione di un resa all'onnipotenza di un nuovo Capitale, più subdolo e proteiforme, quasi lo vanifica, in scherno alle precedenti baldanze utopistiche. In effetti il naufragio degli ideali progressisti-riformistici all'altezza della metà degli anni Settanta, la fuoriuscita dal mondo industriale, la dissidenza dentro la sinistra extraparlamentare prima e di Rifondazione comunista poi, determinano nello scrittore, per una consistente dinamica di contrappassi ideologici, una notevole inversione di rotta della sua traiettoria narrativa e una crescente estraneità alle contemporanee forme del postmoderno, inclini all'idoleggiamento del pastiche, ad un ilare cinismo manipolatorio e aideologico. Il profilo del opera di Volponi si risentisce in una isolata eccentricità, veramente inedita e singolarmente, a suo modo, mostruosa. MdC, pubblicato nel 1989 dopo un decennio di lavoro, è, infatti, il libro della negazione sarcastica, del furore, acre nella revisione delle proprie mitologie personali, caustica nell'impotenza.

L'ultimo romanzo di Volponi si fa voce di un violento e struggente rovesciamento parodico di quanto lo aveva preceduto e instaura con i romanzi anteriori un rapporto che, per molti versi, può rimandare pure, nella strozzata prevalenza di un livido grottesco, disperatamente ilare nel capovolgere, irridere, alla relazione che, in Montale, una raccolta come Satura stabilisce con la sibillina conoscitività delle raccolte anteriori. Gli assi portanti dello stile di Volponi subiscono un fosco mutamento di segno di una violenza pari a quella con cui vengono scherniti gli ideali di riformabilità del Capitale. In una struttura globalmente allegorica (su cui hanno insistito soprattutto Luperini, Contini e i saggi contenuti nel volume curato dal Gruppo Laboratorio 1995$)^{2}$ che indica volontà di distanziamento straniato non c'è più spazio per la vocazione utopistica della scrittura e il revisionismo colpisce tutti gli addendi della prosa dell'autore. L'autore punta, scartata l'ipotesi soggestivisticamente selvaggia dell'io allucinatorio, su di una struttura coralmente caleidoscopica, molteplice, con cui mette mano ad un vasto repertorio della tradizione, ma vanificadone le risorse, tramite saturazione, bruciandole nel contatto con la contemporaneità del disincanto postindustriale. L'ambiziosissima rappresentazione della globalità della crisi, del processo degenerativo del mondo occidentale convoca un montaggio multiplo, pluriprospettico, a blocchi, allineativo nell'alternare narrazione epico-burlesca, dialogo filosofico, monologo riflessivo, invettiva, asso-

2. In Serge Vanvolsen, (a cura di), I tempi del rinnovamento, Roma-Leuwen: Bulzoni, 1995, p. 535-547, Gabriella CONTINI,, "L'ultimo Volponi: invenzione e strategia», Allegoria 9, 1991, p. 93-101. Gruppo Laboratorio (a cura di), Paolo Volponi: scrittura come contraddizione, Milano: Francoangeli, 1995. 
lo diaristico, operetta morale, generi comici e ironici, nella sollecitzione di svariati codici espressivi, che contemplano pure cadenze mitico-fiabesche, tratte di realismo, allegorie. Ė come se Volponi saggiasse le potenzialità storiche della letteratura per verificarne la desolata inutilità. Per questo l'apparente intreccio polifonico rinnega le proprie funzioni originarie, quelle di mostrare la varietà dei casi e delle interpretazioni del reale, di dimostrarne l'irriducibilità ad un immagine unica, per tributare, al contario, una sorta di inno capovolto all'identità alienata, di un lirismo sarcastico, con funereo trionfalismo,

Silenzi plastificati. Crepita l'acido cristallino del dominio oligarchico assoluto, narcisistico quanto cinico. Le sue figure sono singolari, altere e preziose, compiaciute e controllate, intelligenti, esatte nei rispettivi posti. Il deposito è filtrato dal pensiero scaldato, dal calore ossigenato, dai desideri illuminati, dal fulgore del successo; è l'universo, l'unico, il solo dell'esistenza e del futuro. Le sue vicende e i suoi moti sono la storia; il suo equilibrio è la natura, la sua salute è la verità, il suo corso la realtà, la sua direzione la ragione, il suo peso la materia, i suoi organi la scienza, il suo sangue l'umanità. La mente e i sentimenti di questo universo sono i loro, di coloro che lo colmano e lo saziano... Altro universo non c'è, nemmeno sottostante e affondata. Nessun altro mondo, nemmeno fra i satelliti spenti degli spazi, né tra le meteoriti attirate da una delle tante orbite. (MdC, p. 34)

Nella diluizione di ogni alterità, pure la tentacolarità di un romanzo che maneggia prospettive plurali per ricondurle ad un unica sola, concentrando l'apparente molteplicità in unità indistinta, non permette di parlare di polifonia: non esiste, infatti, dialettica di punti di vista, pluriprospettivismo, così come non si affaccia nessuna ipotesi di differenza che rende narrabile, affabulabile il mondo rappresentato. E sono vistose le conseguenze di questa falsa pluralita da tutti i punti di vista, dalla scarsa memorabilità dei personaggi, marionette senza storia sopraffatte dall'intercambiabilità delle maschere, delle funzioni; allo scarso coefficciente di narratività di una prosa, in cui l'irrilevanza dell'individuo privato, rotella di un ingranaggio semovente o tassello di una piramide dal vertice invisibile, avvalla e autorizza la latitanza di un intreccio vero e propio: nella grande dispersione centrifuga delle pagine di $\mathrm{MdC}$, nella dilatazione ipertrofica del cumulo oggettuale, le pur numerose storie raccontate non riescono a stagliarsi, rimangono labili, irrilevanti ed evanescenti. Non c'è più spazio, insomma per l'istrionico sublime, esemplare nella sua irriducibilità, del personaggio eversivo: Saluggia e Crocioni, Aspri, paiono ricordi di un altro secolo,

Non ci sono più personaggi perché nessuno agisce più come tale, nessuno ha un proprio copione. L'unico personaggio, è banale dirlo, è il potere. Se ne subisce il clima. (MdC, p. 137)

Nella generale sentenziosità autoriflessiva a piene mani si raccolgono affermazioni che delegittimano l'ipotesi romanzesca, sia decretandone l'inutilità sia 
svelando la natura di un congegno compositivo fondato non sulla progressione diegetica di una vicissitudine umana, ma sull'acronica giustapposizione di lunghe e litanianti descrizioni, a prevalenza disforica, in cui il profilo individuale del personaggio si viene a perdere, in quanto sussunto nella categoria che ne diminuisce il coefficiente affabulatorio,

Niente. Non c’è niente da raccontare. Non si racconta piú. Lo stato procede, si ferma, si corregge secondo la crisi che gli è stata assegnata dall'industria. La crisi delle istituzioni è crisi delle sovrastrutture e delle produzioni. Non c’è proprio niente da raccontare. Non c'è piú Madame Bovary. Ci sono le categorie sessuali, i prodotti farmaceutici, letterari, cinematografici, dietetici, comportamentali. obbligativi. Si poterebbe raccontare come mille mogli tradiscono tutti insieme, sopra la stessa biancheria, i loro mariti appesantiti dal lavoro e dall'ingenuità? I giocatori, i ribelli, gli assassini, i pescatori, i ricchi, gli avari, gli incapaci, sono ormai a milioni, tutti uguali nel mondo. (p. 123)

Sono queste le motivazioni interne del passaggio da un realismo visionario espressionistico che appoggiava la propria struttura sulla narrabilità di un vissuto eretico, ad un realismo allegorico che constata la pervasività di un potere omolgante, che azzera e livella. Nell'aspirazione ad un'avvolgente totalità rappresentativa che abbracci il molteplice caos massmediale, ultratecnicizzato, mosso dall' etica del profitto e dello sviluppo tecnologico automatizzato e indiscriminato si sommano in reciproca coabitazione e dipendenza due crisi (l'osservazione è di Miceli Jeffers) ${ }^{3}$ da una parte la constatazione attonita, sconfortata e acre della crisi dell'individuo della società ipertecnologica, dall'altra quella della sua rappresentabilità narrativa. Da questa duplice ragione discendono da un lato la dominante paratattico-descrittiva, nel piccolo e nel grande, di un montaggio che non gerarchizza ma aggrega, complice l'intercambiabilità di tutto con tutto, nell'assenza di progressione, dall'altra le copiose schermaglie dialogiche, metalinguisitche e metalettarie, che saggisticamente arrestano il flebilissimo intreccio, la peripezia inconsutile. Dalla falsa ricchezza dell'uniforme consegue sia la crisi della narrativa e dell'intreccio sia la critica dall'interno che origina una sorta di controrappresentazione.

Sparita la prospettiva critica del personaggio unico, l'espressività visionaria, che faceva corpo con esso, a tenere il campo, ad essere personaggio centrale è la Mimèsi del discorso aziendale e del magma dei gerghi diplomatici, ideologici e settoriali, nella proliferante germinazione cancerogena di un discorso identico che fluisce attraverso la voci dei vari attori. Non, si badi, una lingua individuale, personalmente lavorata, ma la fluente lingua di un coro, di una collettività alienata nella grande Chiacchiera universale (lo spunto è di Fortini). ${ }^{4}$ A farla da padrone è la tecnocraticità di una lingua onnipervasiva, dietro cui, invisibile, inservibile, pulsa, ma ormai murata e inattigibile, la realtà. È come

3. Giovanna MiCELI JefFERS, «Linguaggio settoriale e linguaggio letterario: Le mosche del capitale», Critica letteraria, 73, 1991.

4. Franco ForTINI, «Contro il delirio verbale del potere», L'Indice, 6, 1989. 
se, aggiornatasi, la specie burocratica contro cui nei romanzi anteriori si scontravano le avventure sediziose dei protagonisti, avesse occupato interamente il paesaggio della finzione. La tonalità del antiromanzo è di una ridondante medietà fredda e speciosa, marcata dal tecnicismo finanziario di una prosa che straripa, non espressionisticamente, ma per via di riproduzione ecolalica. Prova ne sia l'assenza di quel lavorio di aggressione e manipolazione morfologica che connotava innanzi inquietudine espressionistica, ribelle tensione, e la predilezione, in questo settore, di formazioni e apporti specialistici, lontani da innovatività, estro, capriccio. Costella il romanzo un vocabolario economico-manageriale, scontato: «tempo-produzione», «sistemi di inquadramento», "plannig», "superelettronico", «feeling goals», "preavviso", "profitto», "praticabilita», «fringebenefits», "partite finanziare», "tassi di interesse», «superattivi», «interrelazioni», "dividendi», «tassi di interesse», «diseconomie». "Mai, come in questo romanzo era apparso così chiaro che il potere è soprattutto potere nel linguaggio e del linguaggio e che esso passa attraverso la sua trasformazione da luogo della espressione e della comunicazione a gergo autistico, a logorrea meccanica autofinalizzata intesa solamente alla propria autoaffermazione» (Luperini nel saggio citato)

...come avrebbe potuto lui scendere dalle parole dell'azienda ancora più fredde e lontane, meccaniche e automatiche... e trovarvi sotto un deposito qualunque di verità socialmente accettabile e anche scambiabile? Lui aveva davvero l'impressione di camminare, vivere e lavorare soltanto sulle parole, sui termini di relazioni, dichiarazioni, principi, ordini e organigrammi. (p. 216) Si aspettava da un momento all'altro l'uscita dalla villetta presidenziale, immersa tra gli alberi... del gruppo del consiglio d'amministrazione, riunitosi d'urgenza in seduta straordinaria e deliberante, completa di notaio scrutatore... (p. 181-182) Si vantarono di quella severità distintiva ed esemplare, tecnica come politica, da società industriale avanzata e moderna, sicura sul presente come ben lanciata su un futuro di scienza e di programmazione socio-economica, eco-politica. (p. 26)

In questo contesto le figure della compresenza, della correzione, che nel saggismo dei romanzi anteriori erano veicolo di differenziazioni saggistiche, qui segnalano piuttosto le equivalenze di una verborrea fine a se stessa, ironicamente mimata. Il primum del romanzo sembra proprio essere l'imitazione critica, satirca, della strarpiante chiacchera di un potere autocelebrantesi attraverso un unico discorso in cui i singoli non sono che rotelle di una voce unica,

Gli danno presto la parola ed egli imposta con precisione ed umiltà, a mezzi toni, ragionando su ciò che è evidente e noto, anche se non calcolato e guidato, sia nella città corpo urbanistico, storia e vicende, che del rapporto obbligato, automatico, quanto totale, non considerato quanto corrente e decisivo. (p. 213)

Su questa via si giunge a protratti esiti ecolalici, in cui l'ostinata insistenza su identici ritmemi e fonemi conduce a cadenze cantilenanti, 
...reagiva, parlando serrato e a lungo, con una corrispondente opposta dovizia di particolari, ma molto automaticamente dentro alle consonanze e agli accenti, quasi seguitando nell'eloquio l'andamento involuto del manto, mai trovando soluzioni né uscite. (p. 210)

In effetti l'interesse, spesso metalinguistico, per le colate isofoniche, costante dei romanzi anteriori, perdura ma si ribalta il suo senso, che non indica più evasione o buffonesca irriverenza, ma si limita ad irridere i costituenti del linguaggio aziendale, assunto e svuotato dall'interno, nella voce in off del narratore extradiegetico,

...venuta con solerzia e preoccupazione ad accertare se vi fosse in quella decisione qualche pericolo più grave, sociale, regionale, occupazionale, ambientale, culturale. (MdC, p. 26) ... per contenere in qualche modo la loro indignazione professionale e anche culturale e anche personale, addirittura nazionale e prima ancora civile, da cittadini liberi e coscienti del mondo occidentale! (p. 173) ... ma addiruttura poteva essere ostentato come indice della riservatezza e della informalità richieste per il colloquio, perfino di una convinta cordialità e di una confidente convinzione nell'opportunità e proficuità dell'incontro. (p. 50) I due non avevano saputo guidare al profitto un'azienda pur floridissima e in forte, favorita quanto favorevole espansione (p. 26) Il Presidente impedì ai due amministratori di indire una riunione di chiarimento, di informazione, di giusta partecipazione come di motivata e fondata mobilitazione, di tutti i dirigenti. (p. 26)

L'impiego protratto di una figura fonica, omoteleuto, alliterazione, paranomasia che sia, segnale una sovradeterminazione espressiva, nel senso di una cantilena parodica che demistifica i significati sui quali agisce. Il narratore si vuole lontano da come parla, ma la tabe della stereotipia linguistica dilaga. Non è difficile dimostare come le soluzioni di questo registro pervadono l'insieme delle voci di un coro prostrato e adorante: attraverso pappagalli e ficus, terminali e poltrone operai dirigenti costellazioni, nel beffardo animismo del romanzo, si effonde, terribilmente suasiva, la voce di un potere unico. Ad un analogo trattamento iperbolico e vanificante vengono sottoposti tutti i vettori dello stile dell'autore, in potente autoparodia. Se, come recita p. 56 la narrazione è il bancone del supermercato, la scrittura, per lo più, si limiterà ad un inventario sconsolato dell'eterogeneo delle merci, in cui la parificazione dei reali non sarà da attribuire ad una creaturalità che tutto dignifica, e che a tutto da voce e presta ascolto, quanto alla compiuta operazione di annichilente indifferenziazione ad opera del Moloch capitalistico. Se narrare non si può, se i potenziali affabulativi sono ridotti prevarrà l'uniforme tecnica dell'elenco che affastella, spesso in assenza della punteggiatura, che separa, analizza, distingue,

...amministratori delegati dirigenti consulenti assitenti esperti quadri funzionari addetti capi, via via graduati infeudati remunerati complicizzati in teorie 
di sostegno, in corone pendaggli lustri (p. 13) ambizioni e conoscenze abiti camicie cravatte scarpe (p. 6) Le case basse di puttane venditori ambulanti ladri garzoni maschere di cinema trasportatori fattorini vigilantes studenti apprendisti ballerini travestiti indossatori restauratori fotografi piazzisti venditori di enciclopedie confidenti della polizia, questurini galoppini elettorali prepensionati detenuti in liberta ' provissoria testimoni giurati posteggiatori falsari massaggiatori barbieri di cani gatti uccelli sciemmie tartaughe pesci criceti... (p. 18)

Non si contanto le pagine contraddistinte unicamente da lunghi inventari: le enumerazioni caotiche euforiche con cui quel cannibale della realtà che era Aspri triturava e metabolizzava la realtà, redimendone vitalisticamente la carica negativa, si rovesciano in disforiche elencazioni che «distorcono la ricchezza ordinata in caos indifferenziato". ${ }^{5}$ La passione enumeratrice non cerca di spingere le presenze della realta verso la salute del movimento, e nemmeno di travolgere fondendole in una tensione unificante, nell'oggettività di un progetto liberatorio ma si limita ad inserirele in una piramide del potere, coinvolgendo, dai vertici ai minori, oggetti figure persone della contemporaneità distorta: si tratta di un capovolgimento che è anche intensificazione vanificante,

Dorme la stazione ferroviaria, dormono anche le farmacie notturne, le porte e le anticamere del prontosoccorso, dormono le banche: gli sportelli le scrivanie le poste pnuematiche le grandi casseforti i locali blindati; dormono l'oro l'argento i titoli industriali... (MdC, p. 5)

L'accumulazione da figura della passione per il reale passa ad essere regstrazione constatativa del Capitale onnipotente. La scrittura inventaria l'accumulazione delle merci, nomina uno ad uno i sottoposti al grande capitale, registra il livellamento e l'uniformazione. In tal modo si configura la mappa di un potere (cfr. p. 255-6) dilatato, uno spazio intriso della forza omologante del capitale che irrigimenta i propri sottoposti: l'uso frequente, infatti, del plurale globalizzante, spesso retto da quei principi fonici constatati, certifica la dissoluzione dell'individuale, del singolare caso che contesta. Non sorprende che in uno scenario siffatto le punte sentenziose e oracolari che nei romanzi precedenti si coagulavano in deprecazioni minacciose, in apocalittiche apostrofi ammonitrici, in MdC precipitino nell'urlo strozzato e desolato di una negatività anaforicamente ribattuta. Di nuovo è efficace un confronto,

No officina né fabbrica né industria né città, no orizzonte no paesaggio nemmeno viaggi, no case no strada no tram, niente treno né corriera, nemmeno la vecchia miseria, no trasferimenti né turni, no cielo no faccia no acqua no libro no macchina no ferro no maglia no cintura no tasca no cazzo, più paura no carezze no parole no balli né partite, no ferie no paese no saliva, nemmeno

5. Pier Vincenzo Mengaldo, Storia della lingua italiana. Il Novecento, Bologna: Il Mulino, 1994. 
ingoirae no no fieri nemici. (MdC, p. 211) Un giorno verrà, verrà, ti assicuro che verrà anche se potranno essere molti, molti treni. Sta arrivando per me, che mi porta via, che mi libererà da questa armata di balle. Che esercito: che messe di menzogne, signori! In piedi, correte alla finzione; riverite la menzogna. Sarebbe facile farsi cullare, portare avanti da questo mare di balle, verso le nostre rive. $\mathrm{Ma}$, io mi rifiuto; cerco una rotta diversa, fuori dal fiato e dai piedi della grande armata: signori ufficiali, signori dirigenti, signori aspiranti! E allora vado da solo e voi mi deridete e soffiate contro la mia vela perché il vostro mare di balle la sommerga. (Co, p. 727)

Nella satira che decostruisce, decompone con furore di totale negazione sulla società contemporanea sotto il domino del capitale finanziario, nella devitalizzata congerie oggettuale che stipa una scrittura aggressiva, non sorprende neppure che la logica disumanizzante nella sudditanza ad un disvalore globale, privi di umanità i personaggi. Questi, in accordo con la costante grottesca irridente introdotta dal titolo, marionette risibili, patiscono l'acuirsi e il dilagare di un registro comico che stravolge l'umano, in tonalità che riprendono quelle omologhe delle opere precedenti, ma che qui acquistano una stridula costanza e intensità,

Tirò via il cannello dal profondo della gola e si accostò il telefono alla bocca; ma dopo aver cercato invano di urlare, si cacciò tutta la cornetta dentro il taglio della gola, e sibilò sforzandosi con tutto il corpo, tanto da sprizzare fuori dalle punte delle dita unghie lunghe e dure come chiodi. Morì schiantando la bocca come un bulldozer governativo colpito dal bazooka di un guerrigliero. Rigettò metà cornetta al di là della caratodie. (p. 277)

L'intuizione marxiana che il Neocapitalismo si manifesti e si voglia come seconda natura, che la sua egemonia si dia a patto di uno sradicamento dell'uomo da stesso, riceve nel romanzo efficace rappresentazione nel dissolversi del paesaggio, tutto di interni, artificialmente connotato,

Clima luci aria odore erano uguali da molto tempo, un tempo fisso dentro la MFM. Cambiava appena l'odore degli ambienti. (p. 181)

In questo modo le precedenti corrispondenze tra io e mondo vengono più che soppiantate dall'estraneità reciproca tra soggetto e oggetto, uomo e natura, da una loro perversa identità: umano, naturale vivono congelati in una spazio e un tempo artificiali,

A qust'ora alle vetrate dei piani piú alti si specchiano, con un bicchieri in mano, i dirigenti, di pesante cristallo. (p. 56)

A questo punto è ovvio costatare che la ribalda corporeità pulsante e simbiotica di prima, è, con un ghignante ribaltamento in cui è difficile non leggere scherno e sarcasmo, attribuita all'Ente che tutto asservisce,

Tale sistema, quindi, altro non avrebbe potuto fare che riconoscere anche lui come parte del corpo e quindi alimentarlo, più che per la sua misura e fun- 
zione, secondo la propia corrente discrezionalità variamente ricca e fluida, coagulata e embolica, anemica o iperlibidica. Il sistema era segreto e naturale, fisico e involontario, circolo e regola della circolazione, portata e distribuzione, recupero e spurgo. (p. 258)

La metaforicità animistico-visionaria attraverso cui nei primi romanzi affiorava la componente panica la dimensione irrazionale, su cui faceva leva allucinatoriamente la eversività della prosa dell'autore, ora connota la pervasività di un unico corpo-mondo: paesaggio naturale e corporeita animale sono azzerati; agisce solo il sistema immunitario, fagocitante e autodifensivo del capitale (devo l'osservazione a Zinato, ${ }^{6}$ il cui saggio cito meno di quanto debba). Fermento e movimento sono apparenti. La vivida materialità degli oggetti, che nella lingua antropomorfica dei primi romanzi, svelava un deposito giacente di energie liberatorie, oramai assorbita, sussunta nella circolazione monteria, nel valorie di scambio. "L’inaudito paesaggio di cui il romanzo è mappa è quello di un Capitale puro che ha ultimato la colonizzazione della Natura e dell'inconscio.» ${ }^{7}$ Non vi è più esaltazione e francescana lode delle cose, della loro corporea vitalità irrazionale, nella dilagante irrealtà smaterialazzata indotta dal capitale finanziario. Le figurazioni naturali in cui nei romanzi anteriori si vagheggiavano antitesi, conciliazioni, proposte, prendono qui una curvatura beffarda, nella generale debàcle dell'utopismo. Decantato e abolito l'io arcaico, irrisa ogni possibilità di trasfigurazione visionaria, idealizzante, ne consegue la riduzione dell'ardore metaforico espressivistico, spesso declinato in timbri elegiaci, nel contrappunto degli a parte monologanti,

Queste vallate... sono davvero la spoglia giacente della sua nostalgia (p. 28) Ieri nessuna impressione gli fece un branco di tordi, proprio sui pali del recinto del giardino, nella casa che abita, parvero fuori posto, inutile capriccio di un tempo perduto, mentre saliva in macchina. (p. 28)

L'idea centrale che la prepotenza del Capitale si manifesti nell'alienazione dei linguaggi pubblici burocratici e tecnici diviene strategia parodica di umiliare il loro realismo dell'irrealtà attraverso l'anacronismo della lingua lirica, mostrando l'insignificanza dell'una e l'inattualità dell'altra, senza che ve ne sia una di vera. Ne nascono analogie ibride, in cui l'eco perduta degli incanti conflitta dolorosamente, in un falsetto parodico, con le immagini del disvalore,

La luna... la luna muta e tradisce, indaga e serve le correnti di tutti, amplia, soffice ed organizzata, capace ed attiva come una finanziaria di Zurigo o uno staff harvardiano di consulenti. La luna fila rapida, gemente satellite della memoria ormai arresa; scorre la graduata traiettoria della sua complicità. A quest'ora schizza di telefonate intercontinentali, seleziona e ribalta i nastri delle telescriventi, rimescola perfino il ghiaccio nei bicchieri, imputa e conferma i pen-

7. Emanuele ZinATO, «Paolo Volponi», Studi Novecenteschi, 43-44, p. 7-50. 
sieri e anche li compone su pellicole dotate della selettività delle immagini e delle memorie... il plenilunio glorioso ruota il suo disco monetario. (p. 56)

La profanazione risulta ancora più eloquente se confrontata con la lontana immagine,

La luna appariva a metà ma un poco frangiata in basso: il suo mento era sfuggente e la sua espressione sacra, con la bocca dilatata immensamente che si perdeva nella risposta di un oracolo per tutto il cielo.

Vi è in definitiva discontinuità nella continuità, o meglio radicalizzazione degli aspetti disforici del lirismo precedente: i tempi cambiano... 